\title{
Does the Coherent Lidar System Corroborate Non-Interaction of Waves (NIW)?
}

\author{
Narasimha S. Prasad* \\ NASA Langley Research Center, 5 N. Dryden St., MS 468, Hampton VA, 23681 \\ Chandrasekhar Roychoudhari \\ University of Connecticut, Storrs, CT 06269
}

\begin{abstract}
The NIW (non-interaction of waves) property has been proposed by one of the coauthors. The NIW property states that in the absence of any "obstructing" detectors, all the Huygens-Fresnel secondary wave lets will continue to propagate unhindered and without interacting (interfering) with each other. Since a coherent lidar system incorporates complex behaviors of optical components with different polarizations including circular polarization for the transmitted radiation, then the question arises whether the NIW principle accommodate elliptical polarization of light. Elliptical polarization presumes the summation of orthogonally polarized electric field vectors which contradicts the NIW principle. In this paper, we present working of a coherent lidar system using Jones matrix formulation. The Jones matrix elements represent the anisotropic dipolar properties of molecules of optical components. Accordingly, when we use the Jones matrix methodology to analyze the coherent lidar system, we find that the system behavior is congruent with the NIW property.
\end{abstract}

Keywords: The NIW property, Coherent Lidar, Polarization, Jones Matrix

\section{INTRODUCTION}

In the absence of any 'obstructing' detectors, all the Huygens-Fresnel secondary wavelets will continue to propagate unhindered and without interacting (Interfering) with each other. Light beams do not interfere by themselves. The superposition effects due to light beams become manifest through the response characteristics of the detecting dipoles. Accordingly, all the properties that we generally attribute to only light, are in reality manifestations of collective properties of dipole-light interactions. "Interference" and "coherence" can be better understood in terms of this mutual interaction, followed by energy absorption by the dipoles from EM wave fields, manifesting in some measurable transformation of the detecting dipoles. The NIW property states that in the linear domain, all waves pass through each other unperturbed [1,2]. Different harmonic undulations of the same tension field cannot exert any force of interaction on each other. Otherwise the above observations would not be possible. Well formed light beams cross each other without modifying each other's spatial and/or temporal energy distribution. EM waves do not interact with each other. We have even named "photons" as Bosons. Figure 1 illustrates four scenarios that acknowledge the NIW-Property of NonInteraction of Waves in nature. In the linear domain, all waves pass through each other unperturbed. Different harmonic undulations of the same tension field cannot exert any force of interaction on each other. Otherwise these observations would not have been possible? In general, we have been ignoring the absence of any physical interaction process (force) between waves! In reality, the subtlety of non-interference of wave forms is a generic property of nature!

Let us now consider the manifestation the NIW property in complex systems. We now ask ourselves "Does complex optical systems having several optical elements manipulating polarization accommodate NIW principle? For e.g., elliptical polarization presumes the summation of orthogonally polarized electric field vectors. Does elliptical polarization contradict the NIW property? We explore the NIW principle using Jones Matrix formulation applied to a coherent lidar system since it uses polarization elements in a distinctive way. In this paper, we show that from Jones matrix analysis, electric fields will not reorganize themselves as elliptically spinning e-vectors. The two orthogonal electric field vectors continue propagating independent of each other through optical components and the media. Jones matrices provide one more means to confirm the NIW property.

\footnotetext{
* narasimha.s.prasad@nasa.gov; Phone 757-864-9403; Fax 757-864-8828.
} 


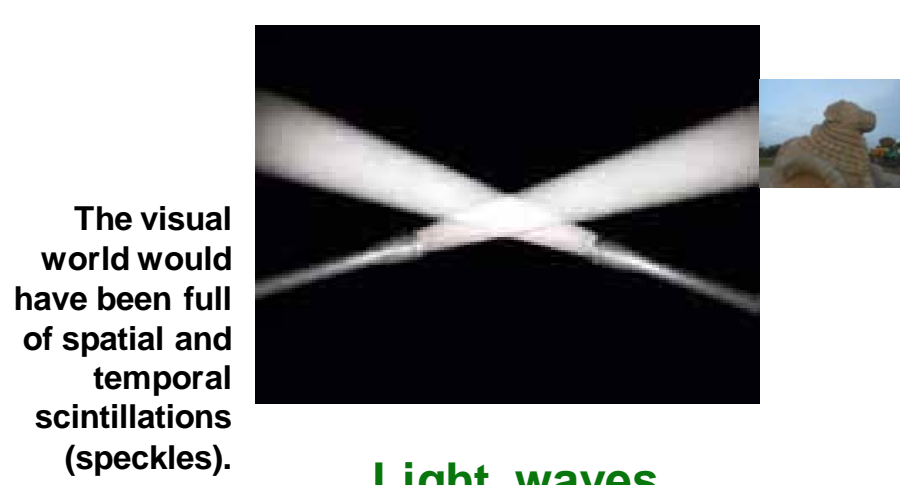

Light waves
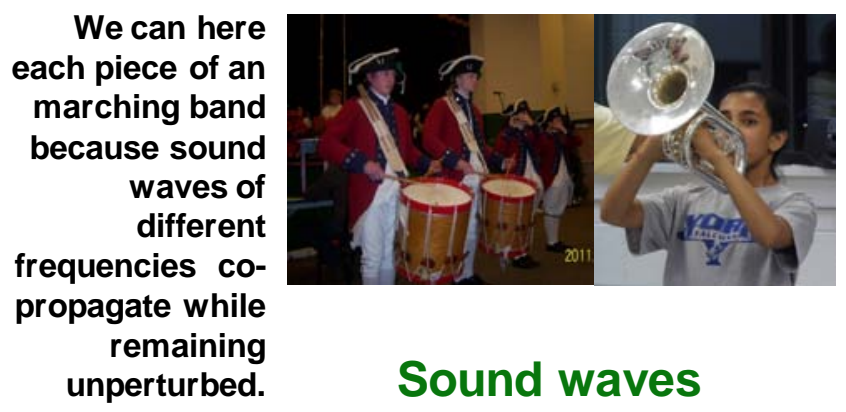

Sound waves

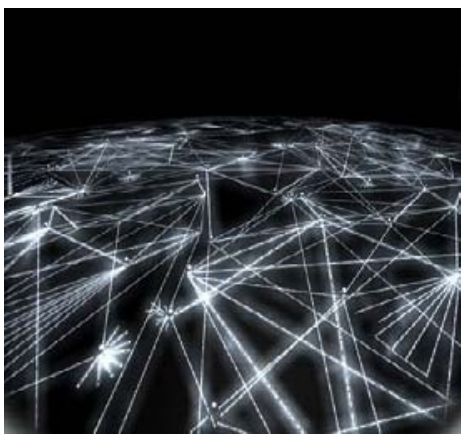

Light waves

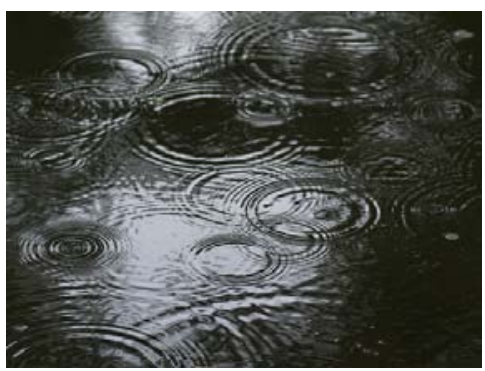

Water waves
WDM

internet data would have been destroyed by temporal interference (heterodyne effect).

Figure 1. Four scenarios illustrating the NIW property. Top left: The fidelity of the visual world remains unperturbed even though all optical beams are always crossed by innumerable other beams. Top Right: Spectral and Doppler shift information carried by any specific star light remains unperturbed by billions of other crossing star lights. Bottom Left: NIW property in the acoustics domain and co-propagation of acoustic waves without interference. We can discern all the different frequencies from different instruments. Bottom Right: The NIW property exhibited by water waves. Water waves pass through each other without perturbing each other's original propagation behavior!

\section{COHERENT LIDAR SYSTEM}

Figure 2 shows the schematic of a typical pulsed coherent lidar system commonly utilized for remote sensing of winds, wake vortices, atmospheric trace gases, and hard targets [3]. If a CW laser is used, an intensity modulator or a pulse slicer would be used. The vertically (or horizontally) polarized laser light is converted into circularly polarized light by using a quarter wave plate at the output end before transmitting it out. Circular polarizing light avoids depolarization issues. The reflected light from aerosols will change its handedness, right to left or vice versa. When the return light is collected by a telescope and passed through the same quarter wave plate, it will be horizontally (or vertical) polarized which will now be separated out using a polarizing beam splitter. The output beam will now be heterodyned (mixed) with a local oscillator signal which is normally derived from the master seed oscillator present in the laser unit by frequency shifting its wavelength by an acousto-optic modulator. The heterodyned signal is now incident on the detector. The detected signal components are later amplified, processed, and analyzed using signal processing electronics. Previously, the heterodyne scheme was investigated for detection requirements [4]. In this paper, functioning of a coherent lidar system is investigated for its compliance with the NIW property. In the following, section, we will use Jones Matrix formulation to illustrate the functioning of a coherent lidar system. 


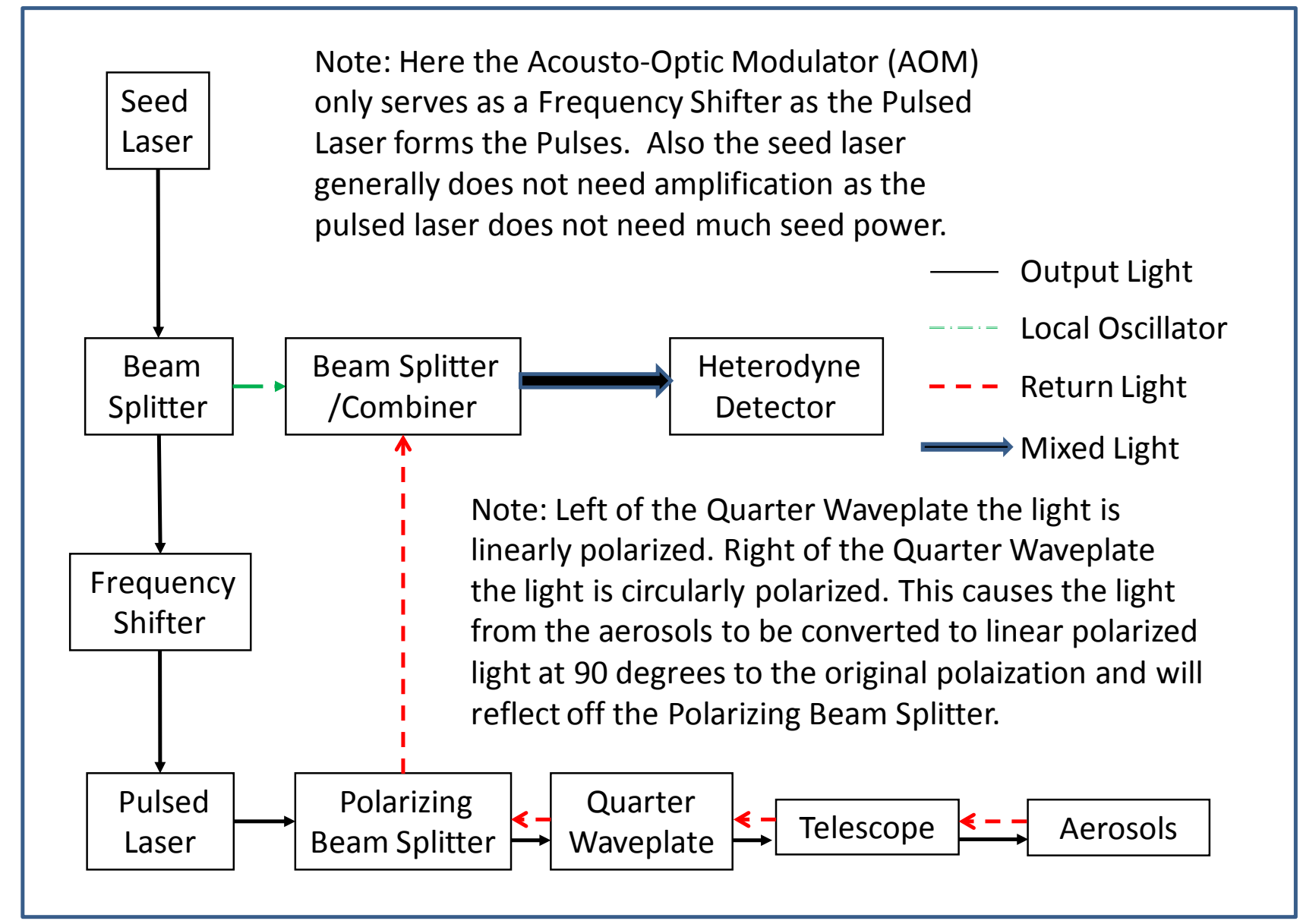

Figure 2. The schematic of a pulsed coherent lidar system used for wind sensing.

\section{JONES MATRIX FORMULATION}

Jones Matrix is a 2x2 matrix methodology to treat polarization state of light as dipolar amplitude induced by corresponding electric vectors [5]. Jones Matrices elegantly keep track of polarization state through polarizing components, their orientations and coordinate frame of references. Also, Jones matrices take care of superposition effects carried out by material dipoles of the polarizing components because they carry out resultant undulations. The dipolarizing molecules in the optical components transmit the resultant amplitude corresponding to each parallel components. In a general case, the resulting Jones matrix is a product of Jones matrix of individual components operated in the consecutive order the light propagates through them and is as shown below:

$$
\left[\begin{array}{l}
\mathrm{E}_{\mathrm{x}}^{\prime} \\
\mathrm{E}_{y}^{\prime}
\end{array}\right]=\left[M_{n}\right] \cdot\left[M_{n-1}\right] . .\left[M_{1}\right] \cdot\left[\begin{array}{l}
\mathrm{E}_{\mathrm{x}} \\
\mathrm{E}_{\mathrm{y}}
\end{array}\right]
$$

The emergent state of polarization of an electric vector due to propagation of an incident vector would be expressed using the Jones' matrix method as , which, in matrix form, can be written as:

$$
\begin{aligned}
& E_{x}^{\prime}=J_{x x} E_{x}+J_{x y} E_{y} \\
& E_{y}^{\prime}=J_{y x} E_{x}+J_{y y} E_{y}
\end{aligned}
$$


For amplitudes, we can now write,

$$
\left(\begin{array}{c}
E_{x}^{\prime} \\
E_{y}^{\prime}
\end{array}\right)=\left(\begin{array}{cc}
J_{x x} & J_{x y} \\
J_{y x} & J_{y y}
\end{array}\right)\left(\begin{array}{l}
E_{x} \\
E_{y}
\end{array}\right)
$$

In Eq. 3, J's represent dipolar stimulation of the polarizing molecules present in the optical component in each of the directions defined by subscripts. Thus the superposition effect at the amplitude state is carried out by the dipoles but not the fields. There are no direct field-field cross terms in the superposition but there are cross terms corresponding to dipolar undulations. This implies fields are not summing themselves (the NIW property).

If we consider intensities, we can write,

$$
\begin{aligned}
& D_{\text {input }}=E_{x}^{2}+E_{y}^{2} ; \quad D_{\text {output }}=E_{x}^{\prime 2}+E_{y}^{\prime 2} \text {. Note: } D_{\text {output }} \neq\left|E_{x}^{\prime}+E_{y}^{\prime}\right|^{2} \\
& D_{\text {output }}=E_{x}^{\prime 2}+E_{y}^{\prime 2}=\left[J_{x x} E_{x}+J_{x y} E_{y}\right]^{2}+\left[J_{y x} E_{x}+J_{y y} E_{y}\right]^{2}
\end{aligned}
$$

From Eq. 4, there are no cross terms in Electric field components. Hence, it can be seen that the NIW property is built into the Jones Matrix formulations. In the next section, we apply the Jones matrix methodology to a coherent lidar system.

\section{JONES MATRIX APPLIED TO A COHERENT LIDAR SYSTEM}

Figure 3 shows the coherent lidar architecture corresponding to Figure 2 illustrating the polarization information of the laser beam as it propagates through major components. Figure 4 shows the components laid out serially as the laser beam propagates from transmitter to aerosols and back to the detector.

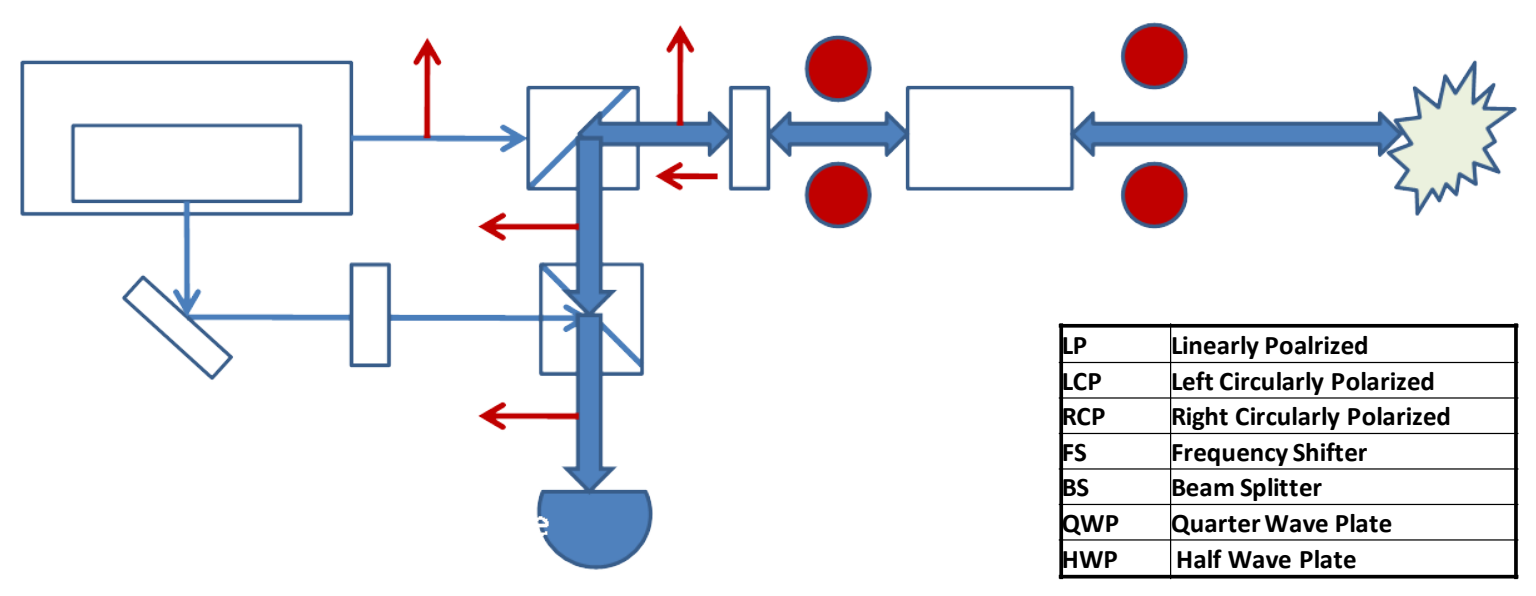

Figure 3. The coherent lidar system illustrating the polarization of laser beam. 


\begin{tabular}{|c|c|c|c|c|c|c|c|}
\hline$[$ Laser $]=$ & [BS] & [PBS] & [QWP] & [Telescope] & [Aerosols] & [Telescope] & [QWP] \\
\hline
\end{tabular}

Figure 4. The coherent lidar components corresponding to Figure 3 in the path of laser beam propagation.

We now write the corresponding Jones matrices in the normalized form for the components indicated in Figure 4. For our analysis, no loss of intensity loss is considered for simplicity purposes.

$$
\begin{array}{r}
{\left[\begin{array}{l}
\mathrm{E}_{\mathrm{x}}^{\prime} \\
\mathrm{E}_{y}^{\prime}
\end{array}\right]=\left[\begin{array}{ll}
1 & 0 \\
0 & 1
\end{array}\right]\left[\begin{array}{cc}
\cos \theta & -\sin \theta \\
\sin \theta & \cos \theta
\end{array}\right]\left[e^{-i \pi / 4}\right]\left[\begin{array}{ll}
1 & 0 \\
0 & i
\end{array}\right]\left[\begin{array}{cc}
\cos \theta & \sin \theta \\
-\sin \theta & \cos \theta
\end{array}\right]\left[\begin{array}{ll}
1 & 0 \\
0 & 1
\end{array}\right]\left[\begin{array}{ll}
1 & 0 \\
0 & 1
\end{array}\right]\left[\begin{array}{ll}
1 & 0 \\
0 & 1
\end{array}\right]} \\
{\left[\begin{array}{cc}
\cos \theta & -\sin \theta \\
\sin \theta & \cos \theta
\end{array}\right]\left[e^{-i \pi / 4}\left[\begin{array}{ll}
1 & 0 \\
0 & i
\end{array}\right]\left[\begin{array}{cc}
\cos \theta & \sin \theta \\
-\sin \theta & \cos \theta
\end{array}\right]\left[\begin{array}{ll}
1 & 0 \\
0 & 1
\end{array}\right]\left[\begin{array}{l}
0 \\
1
\end{array}\right]\right.}
\end{array}
$$

Upon simplification, Eq. 5 reduces to

$$
\left[\begin{array}{l}
E_{x}^{\prime} \\
E_{y}^{\prime}
\end{array}\right]=\left[e^{i \pi / 4}\left[\begin{array}{cc}
1 & 0 \\
0 & -1
\end{array}\right]\right.
$$

Hence, ignoring any loss mechanisms due to absorption or misalignment, the coherent lidar system effectively reduces to a HWP from the stand point of polarization analysis.

\section{SUMMARY AND CONCLUSIONS}

In this paper, the NIW property is investigated in regard to a coherent lidar system using Jones matrix formulism. The objective is look into optical systems that may violate NIW property. Validation of the NIW property for various optical systems is essential for its recognition as a basic property of propagation of photons. For our simplified analysis, a generic coherent lidar system architecture is considered. From the Jones matrix formulism, we see that the J's are the material oscillatory properties. Cross terms in the J's represent the superposition effect carried out by the dipoles through their simultaneous stimulations due to projected components of the orthogonal fields. There are "no field-crossterms" in the Jones matrix analysis. Thus Jones matrix formulism for a coherent lidar system validates the NIW property. Further investigation will be into applying the NIW property in detailed system analysis for performance enhancements.

\section{REFERENCES}

[1] Chandra Roychoudhari, “Principle of non-interaction of Waves,” Journal of Nanophotonics, Vol. 4, 043512 (2 July 2010).

[2] C. Roychoudhuri, Causal Physics: Photon Model from Non-Interaction of Waves, CRC, Oct.2013.

[3] T. Fujii and T. Fukuchi, (ed.), Laser Remote Sensing, Taylor and Francis, New York, 2005.

[4] C. Roychoudhuri and N. Prasad, “A deeper look at the fundamentals of heterodyne detection requirements”; Proc. 14th Coherent Laser Radar Conference, Snowmass, Colorado; July 8 -13, 2007.

[5] Jones, R. Clark (1941). "A new calculus for the treatment of optical systems, I. Description and Discussion of the Calculus". Journal of the Optical Society of America 31 (7): 488-493; Also see Hecht, E. (2001). Optics (4th ed.). 